\title{
Docencia universitaria y estudiantes con discapacidad: Cuestiones sobre accesibilidad y adaptación en el estudio.
}

\section{University teaching and students with disabilities: Questions about accessibility and adaptation in the study.}

\author{
Diego Jesús Luque Parra', María Jesús Luque Rojas ${ }^{1}$, Eduardo Elósegui Bandera” Dolores Casquero Arjona ${ }^{1}$, \\ Lourdes Mara llizástigui del Portal ${ }^{2}$ \\ Universidad de Málaga ${ }^{1}$, España, Universidad Santo Tomás, Chile ${ }^{2}$
}

(Rec: junio de 2018 - Acept: mayo de 2019)

\section{Resumen}

Este trabajo aborda la discapacidad en el contexto universitario, desde la adecuación a las necesidades y características de la persona. Esta mirada busca que quienes presenten necesidades especiales asociadas a la discapacidad, dispongan de los medios, apoyos y recursos suficientes para asegurar la igualdad real y efectiva de oportunidades dentro de la comunidad universitaria. De acuerdo con ese objetivo, con este artículo se pretende generar una reflexión en el docente universitario, sobre las cuestiones de accesibilidad y adaptación -como aspectos básicos- en la atención a su alumnado con discapacidad. Estos aspectos se relacionan con la búsqueda de desarrollo tecnológico, formativo y personal-social, propio de una Universidad abierta a la sociedad, apuntando hacia valores de normalización, integración e inclusión. En una primera parte, se tratan generalidades sobre el desarrollo tecnológico y usuarios con discapacidad, para pasar a una segunda en la que se abordan cuestiones de la accesibilidad y la discapacidad, continuando con su concreción en el currículum universitario del alumnado con discapacidad.

Palabras clave: Accesibilidad, estudiantes con discapacidad, tecnología educativa, docencia universitaria, adaptación curricular.

\begin{abstract}
This work addresses disability in the higher education context, in terms of reasonable and achievable adjustments related to the individual's needs and features. This approach aims to ensure assistive technology, support, and resources as means to guarantee real and effective access to equal opportunities for those members that may present special needs, due to their disability situation, within the university community. Therefore, this paper attempts to generate a reflection for university lecturers about accessibility and adaptation, as basic aspects in their duty of supporting disabled students. These aspects are directly related to technological, educational and socio-personal development, present in those higher education institutions open to society and oriented to values such as integration and inclusion. In the first place, some general information is provided about technological development and users with disabilities. In the second place, certain aspects related to accessibility and disability are addressed, and their materialization in the university curriculum of disabled students.

Keywords: Accessibility, students with disability, educational technology, university teaching, curriculum adaptations.
\end{abstract}

Correspondencia a: Diego Luque, dluque@uma.es 


\section{Introducción}

En una sociedad del conocimiento, abierta y democrática, la educación se orienta hacia la construcción de valores para el crecimiento personal y social de los ciudadanos, aspirando a procesos de cambio social y económico que, en suma, favorezcan un progreso humano. En este proceso, la aplicación de nuevas tecnologías en el contexto de una economía globalizada e interconectada, debe contribuir a generar una sociedad más dinámica y solidaria, a la vez que exigente en la formación y preparación de sus ciudadanos en su afrontamiento vital. Tales aspectos, nos llevan a planteamientos como una mejor distribución de bienes y servicios y una política social justa, de forma que, ese progreso humano, alcance a todos los grupos que forman la diversidad de su sociedad (Rodríguez de las Heras, 2006).

En efecto, desde una sociedad del conocimiento, debe admitirse que ese conocimiento es un núcleo de confluencia de la tecnología, la educación y la cultura propia de la sociedad, lo que supone su transformación global afectando, como expresa Rodríguez de las Heras (2006), al desarrollo sociocultural. Cuando esto se valora en el ámbito educativo y se integra en el currículum de programas y actividades de enseñanza, se consiguen objetivos de calidad y equidad en la educación, consiguiendo desarrollo de una vida autónoma, tanto en lo académico, como en lo personal y social.

Para la consecución de tales fines, deberá considerarse un marco de valores y actitudes favorables a la diferencia y la diversidad, así como la disponibilidad de recursos y el diseño de contextos socioeducativos que permitan que el estudiante asuma su responsabilidad en la ejecución de tareas y en el mejor manejo de situaciones. En este sentido, cada educando deberá ser consciente de que su desarrollo profesional dependerá de su quehacer personal y no tanto de las actuaciones del profesorado y de la institución. En otras palabras, su aprendizaje será fruto de su voluntad y desarrollo de habilidades, en conexión con el esfuerzo de docentes y del centro formativo.

Esto que parece obvio para cualquier estudiante, adquiere una especial relevancia en las personas con discapacidad, impulsando su realización personal y la participación activa en la vida de la comunidad. En efecto, para el desarrollo de estas personas es fundamental la acción coordinada, coherente y congruente del profesorado, dentro de un marco de reflexión docente y de acción tutorial sobre sus estudiantes.

Expresar que las universidades se dirigen hacia la excelencia, es aceptarlas como un motor sociocultural y económico de sus regiones, que persigue fines y objetivos de desarrollo. Esto lo hace a través de una educación de calidad para formar a sus estudiantes, teniendo en cuenta las necesidades particulares y la funcionalidad de ellos. Así, deben favorecerse procesos de formación ajustados al alumnado, poniendo en realidad el principio de igualdad de oportunidades. En consecuencia, la excelencia de toda universidad debe apreciarse desde otros parámetros que incorporen la adecuación a diversas situaciones sociales, dando respuestas, tanto desde la accesibilidad y la adaptación, como de la comprensión y el apoyo a cualquier persona que lo precise en sus diversas circunstancias. En este sentido, la Universidad no es (o no debe ser) ajena a la realidad que tienen los alumnos y alumnas con discapacidad. Para ellos, la enseñanza superior supone la continuidad de una educación secundaria post-obligatoria en una formación integral, y no exclusivamente académica o profesional, que implica quizá algunos matices diferenciadores del resto de compañeros sin discapacidad.

Considerando estos antecedentes, en este documento se hacen algunas observaciones sobre las TIC en relación con las personas con discapacidad, buscando la reflexión en el docente universitario, sobre la accesibilidad y la adaptación, como aspectos básicos, en la atención a su alumnado con esa particularidad, todo ello, en dirección hacia valores de normalización e integración, dentro de un marco de inclusión. En una primera parte se tratan generalidades sobre el desarrollo tecnológico y usuarios con alguna limitación, para pasar a una segunda en la que se abordaría la cuestión de la accesibilidad y la adecuación a la diversidad, finalizando con la accesibilidad y adaptación del currículum universitario para el alumnado con discapacidad.

\section{Desarrollo tecnológico y personas (usuarias) con disca- pacidad.}

Puede tenerse en cuenta, a modo de reflexión previa (Luque y Rodríguez, 2009), cómo el desarrollo tecnológico no ha considerado en su origen, las especiales circunstancias de grupos o individuos que pueden tener dificultades de acceso o de aplicación. En este sentido, y sin entrar en un análisis de las causas, puede observarse cómo las personas con discapacidad deben realizar un mayor esfuerzo de superación de sus propias limitaciones, en una sociedad que debe aportar soluciones a la integración de todos sus miembros y especialmente, a quienes tengan riesgo de exclusión. Esta razón apoya que deban proseguirse las medidas políticas y sociales para reducir la brecha diferencial de este sector de la población.

En este sentido, el desarrollo tecnológico debe partir y desarrollarse sobre técnicas y recursos con patrones de diseño universal, evitándose barreras de comunicación, de información o de accesibilidad. En efecto, como han expresado algunos autores (Grau, 2004; Luque y Rodríguez, 2006), a las personas con discapacidad se les debe aportar productos y servicios siguiendo pautas que se adapten a los requerimientos de limitación de habilidades y capacidades, esto es, las llamadas Diseño para todos o Diseño universal y las denominadas Ayudas técnicas (Luque-Rojas, Calvo, Moreno y Luque, 2010). El Diseño para todos persigue el uso de estrategias para diseñar entornos, productos y servicios, que puedan ser utilizados por el mayor número de personas, sin necesidad de adaptación o diseño especializado (cuadro 1 de principios de aplicación a tecnologías y servicios). Por otra parte, las Ayudas técnicas permitirían diseñar productos y servicios específicos en aquellos casos concretos donde la reducción de habilidad o capacidad de manejo del producto no ha sido oportunamente abordada desde el Diseño universal. 
Tabla 1. Principios fundamentales del Diseño para todos

Uso equitativo:

Flexibilidad de uso:

Uso simple e intuitivo:

Información perceptible:

Tolerancia al fallo:

Menor esfuerzo físico:

Tamaño y espacio

adecuados para su

aproximación y uso: el producto debe ser útil para cualquier tipo de usuarios. Debe evitarse la segregación y estigmatización de los usuarios.

el diseño debe adaptarse a la amplitud de preferencias y capacidades individuales. miento, habilidades o nivel de concentración.

el diseño debe comunicar la información perceptible al usuario de manera efectiva, independientemente de las condiciones ambientales o de su habilidad es sensoriales.

minimizar los peligros y las consecuencias negativas de acciones erróneas o accidentales del usuario.

el diseño se debe poder utilizar de manera eficiente, cómoda y con el esfuerzo mínimo posible.

se debe facilitar el espacio adecuado para acercarse, alcanzar, manipular y utilizar el diseño, independientemente del tamaño del cuerpo del usuario, su postura o movilidad.

Nota. Tomado y adaptado de Rose, D. y Meyer, A. (2000). Universal Design for Learning.

Estos principios resguardan que cualquier persona con discapacidad tenga aseguradas las condiciones de acceso y uso de las tecnologías con objetivos de desarrollo personal y social. Ambos tipos de estrategias (diseño universal y ayudas técnicas), son de aplicación en todas las discapacidades. Se puede concluir, que la tecnología implica el diseño, desarrollo y ejecución de herramientas, técnicas o dispositivos orientados al desarrollo individual y socio comunitario, así como a la educación, formación, salud y bienestar de la persona.

\section{Discapacidad y accesibilidad en el estudio.}

Dentro de la Clasificación Internacional del Funcionamiento, la Discapacidad y la Salud, la discapacidad es valorada como circunstancia de aspectos negativos de la interacción del individuo y sus factores contextuales, limitaciones de la actividad y restricciones de la participación (CIFDS, OMS, 2001). Vendría a colación recordar a Vygotsky (ya en su Fundamentos de la Defectología, 1924-1929/1989), quien presentaba una nueva concepción de los procesos de aprendizaje y desarrollo de las personas con discapacidad. En efecto, para Vygotsky, el defecto primario es aquel de naturaleza biológica, diferente del defecto secundario que se origina a partir del primero y considerando la forma en que está estructurado el contexto del individuo, con la inadecuación de los procesos educativos a las necesidades especiales originadas por el defecto primario. Para este autor, el defecto primario no necesariamente implica una deficiencia, sino lo que actualmente podría decirse como una inadecuación del sistema o del contexto. En suma, nos distanciamos así de una concepción médica o anclada en la deficiencia, ya que el defecto no determina linealmente el desarrollo, pudiendo asumir formas diversas en función de los contextos culturales y socio - relacionales, en los cuales las personas con discapacidad participen (Ilizástigui, 2010).

Considerando lo anterior en un ámbito educativo (y en el contexto universitario en particular), la discapacidad (como es- tado o circunstancia) se ha de valorar desde las necesidades y características de la persona, a fin de reducir, compensar o neutralizar con medidas y recursos sus limitaciones o dificultades. En cualquier caso, es necesario centrar el análisis en el sujeto en su integridad, especialmente, en lo que el estudiante tiene y en relación a lo cual pueden ser estructuradas estrategias educativas que contribuyan a promover su desarrollo y aprendizaje. Esta atención además, de forma concreta y operativa, deberá estar recogida en la normativa legislativa para que cualquier universidad pueda favorecer acciones y disponer de los medios, apoyos y recursos para sus estudiantes con discapacidad, asegurando la igualdad real y efectiva de oportunidades, en relación con los demás componentes de la comunidad universitaria (en España, regulado por la Ley Orgánica 4/2007, de 12 de abril, por la que se modifica la Ley Orgánica 6/2001, de 21 de diciembre, de Universidades; en su disposición adicional vigésima cuarta, de la inclusión de las personas con discapacidad en las universidades).

Situarnos en un marco de inclusión y adoptar una concepción abierta e integradora respecto a la enseñanza y al diseño de métodos, servicios o dispositivos dentro del diseño universal, conlleva a posicionarnos en una perspectiva de aceptación de la diversidad social y, consiguientemente, de incrementar la participación de las personas con discapacidad en nuestra sociedad, comunidad o ámbito educativo. En efecto, la propuesta de diseño de productos, servicios y entornos de fácil uso para todos (desde su inicio y sin necesidad de readaptación o nuevo diseño de forma especial), persigue objetivos de simplificación en la realización de las actividades cotidianas, haciendo uso de entornos, servicios o productos sencillos y de menor esfuerzo, con el beneficio para todas las personas, edades y habilidades.

En este marco de un diseño para todos, las TIC proporcionan en los ámbitos educativos, un enfoque de enseñanza-apren- 
dizaje resultante de la investigación y los avances tecnológicos sobre el aprendizaje, respondiendo adecuadamente a las diferencias individuales (Rose y Meyer, 2000; Rose, Meyer e Hitchcock, 2005; Observatorio Universidad y Discapacidad, 2012; Diez y Sánchez, 2014; Rivero-Hernández, 2014). Con ello, puede desarrollarse un currículum y metodologías que se adapten a las características del alumnado, a la vez que se reduce la necesidad que el profesorado realice adaptaciones curriculares a posteriori, proporcionando alternativas en recursos, contenidos, contextos o tecnologías que benefician a todos los alumnos y no solo a los que presentan discapacidad. Ciertamente, esta época de transformaciones y cambios requiere de una dirección y de estrategias que permitan aprovechar al máximo los potenciales de las personas y de las instituciones (véanse algunas sugerencias en el Anexo 1).

Se puede expresar que los aspectos del diseño universal son conocidos y asumidos por la comunidad universitaria, sobre todo en su vertiente arquitectónica y en algunos servicios; no obstante, no es así en lo que respecta a diseño curricular, información y comunicación, de acuerdo al propio núcleo del diseño universal. En estos aspectos, la Universidad a través de programas de atención a universitarios con discapacidad, debe perseguir objetivos de acceso igualitario, servicios equitativos y eficaces, cumpliendo así estándares de calidad (Alonso y Diez, 2008). Con todo, debe considerarse la consecución de dos grandes objetivos de desarrollo en el alumnado (Luque y Luque-Rojas, 2015):

- Potenciar y desarrollar las capacidades, generando autonomía, independencia y acceso a los servicios.

- Compensar limitaciones y mejorar los aspectos de participación e interacción sociales.

De acuerdo a esos objetivos, debe expresarse la conveniencia de estudiar las características de la tecnología, a la vez que las características de la persona y su discapacidad, para evitar el riesgo de barreras de acceso de esa tecnología a su vida particular y cotidiana. Esto es, la tecnología, y sus tecnologías específicas, deben desarrollarse en términos de respuesta a las necesidades especiales que plantean los alumnos y alumnas, a través de los recursos y servicios, adaptándoles los elementos no accesibles o de menor uso, y tratando de desarrollar una vida autónoma, en lo académico, personal y social (Sánchez-Montoya, 2002; Luque y Rodríguez, 2006). En este sentido, como se ha citado por algunos autores (Sánchez-Montoya, 2007; Luque y Rodríguez, 2006, Moriña-Diez, López, Melero, Cortés y Molina, 2013), las TIC deben poner su acento en las destrezas y habilidades que puede desarrollar el estudiante, y no en sus limitaciones, generando un marco en donde pueda aprender a manejar sus dificultades.

\section{Accesibilidad y adaptación del currículum al alumnado universitario con discapacidad.}

Cuando el profesorado universitario atiende a las necesidades de su alumnado en general, y a las del que presenta discapacidad en particular, lo hace aportando los elementos de formación y de trabajo de sus asignaturas, considerando aspectos de accesibilidad y de recursos y ejercitándose en una práctica docente justa y adecuada, en consideración con las características del alumnado, dentro de valores de igualdad, cooperación y apoyo entre personas. En un entorno social universitario de este tipo, en el que se favorece un nivel de autorrealización, cabe señalar que el estudiante con discapa- cidad, de acuerdo con el principio de igualdad de oportunidades, no debe recibir un trato diferenciador respecto a los demás alumnos, sino el de una respuesta compensadora, en su acceso a la enseñanza y formación.

Desde este punto de vista, el sistema universitario es entendido como estructura y organización de provisión de servicios al alumnado, sobre una concepción de accesibilidad, destinada a la atención y respuesta a las necesidades (Alonso y Díaz, 2008; Luque y Rodríguez, 2005). En este sentido no estaría de más expresar que, tanto los recursos en general, como las TIC en particular, son muy bien valorados por el profesorado, aunque el incremento de la disponibilidad de los recursos tecnológicos en los centros educativos no haya supuesto una alteración significativa en la práctica pedagógica o de los modelos de enseñanza tradicionales (Suriá, 2011). En cualquier caso, aceptando que no es tanto la falta de recursos, como la falta de preparación y de actitudes lo que más incide en la enseñanza y en el uso de la TIC en la docencia con alumnado con discapacidad, debería considerarse la búsqueda de un equilibrio entre el saber hacer docente y la tenencia y uso de los recursos, aportándose la base para una evolución y rendimiento académico apropiados, en el alumnado en general y en el de discapacidad, en particular (Luque y Luque-Rojas, 2012).

El proceso de enseñanza-aprendizaje en el nivel universitario, precisa de un currículum que podría definirse como un proyecto formativo integrado (Zabalza, 2003), esto es, un conjunto estructurado de elementos (actividades, metodología, contenidos, objetivos, recursos, etc.), organizado y formalizado, que busca la mejor preparación y formación del alumnado que participa. Ahora bien, un currículum respecto al alumnado con discapacidad, requiere contemplar y compensar sus necesidades, proveyéndolas de los recursos y servicios oportunos, en la línea de accesibilidad y adaptación de los estudios anteriores. Obviamente el currículum universitario es altamente específico por sus contenidos, objetivos y los fines de alta cualificación, tanto en lo formativo y cultural como en la preparación y capacitación profesional de sus enseñanzas (Luque, et al., 2014; Herradón, Blanco y Pérez-Juste, 2009). En este sentido, la formación universitaria para estudiantes en situación de discapacidad, no va a diferir con significación respecto al resto de alumnos, aunque deban hacerse algunas consideraciones en su docencia:

- Reflexión oportuna en el profesorado, sobre el equilibrio entre las condiciones de accesibilidad general (arquitectónica y de servicios) con las propias de la tecnología aplicada a la educación y las consiguientes adecuaciones del currículum.

- Currículum complejo, con diversidad de áreas de conocimiento, diversidad de asignaturas, especificidad de contenidos y objetivos particulares, es decir, un currículum heterogéneo, con una estructura y organización descentralizada, coherente con unas áreas de conocimiento y de carácter terminal (diferente al sistema educativo no universitario, de carácter propedéutico).

- Un diseño curricular (como instrumento orientador de la acción formativa que el docente ha de desarrollar) que, aunque con las características de troncalidad, optatividad y singularidad de los centros universitarios, debe observarse con carácter individual para cada área y asignatura. 
- La conveniencia de llevar a cabo alguna adaptación curricular, es decir, cualquier acción docente facilitadora y de ajuste de las asignaturas para el estudiante que la precise, convirtiéndose en un mecanismo de accesibilidad y de nivelación. Es, en consecuencia, una medida que demuestra la existencia de un diseño curricular para todos, incluida la persona con discapacidad.

Desde estas consideraciones, el profesorado se acerca a la realidad de sus estudiantes, siendo la adaptación un mecanismo de respuesta a las necesidades de formación, a través de las modificaciones precisas en el currículum. El ámbito universitario difiere de la educación secundaria en que los objetivos y contenidos no nucleares no pueden ser alterados (con algunos matices considerando el principio de ajuste), ya que las instituciones universitarias tienen la responsabilidad social de formar profesionales con competencias acordes a su perfil profesional, con planes de estudio orientados al desarrollo y adquisición de los conocimientos y destrezas asociadas a la profesión (Escudero, 2008; Perrenoud, 2008; Herradón, Blanco y Pérez-Juste, 2009; Nava-Caballero, 2012).

La adaptación no afecta a los objetivos, los que se formulan en determinación a los contenidos y competencias necesarios de desarrollar; en consecuencia, la significatividad no será entendida por la reducción o eliminación de objetivos, ya que alteraría la calidad de los núcleos de los planes de estudio, que, a su vez, han de garantizar la competencia profesional (Rodríguez y Luque, 2006; Rodríguez-Martín y Álvarez-Arregui, 2014). Estaríamos en una visión de adaptación curricular, más en accesibilidad y modificación de determinados aspectos curriculares, no implicando la eliminación de objetivos, sino un cambio de dirección en los mismos.

Por todo lo anterior, la adaptación curricular universitaria resulta en una respuesta formativa a través de la accesibilidad y recursos, así como en lo curricular de procedimientos, metodología, evaluación y estrategias de enseñanza-aprendizaje (Luque, et al., 2014).

\section{Discusión}

De acuerdo a los párrafos precedentes, y dado que la discapacidad no determina la formación del estudiante, sino que implica una atención adecuada a sus necesidades y funcionalidad, podría concluirse en que una integración tecnológica-educativa tiene su pleno sentido en la Universidad. En efecto, contribuye a la normalización e integración como auténticas vías de desarrollo personal, cultural y formativo, proporcionando herramientas que desarrollan habilidades y destrezas, favoreciendo la cooperación y ayuda entre los compañeros de aula.

Desde esta perspectiva, las TIC hacen que la discapacidad no se aprecie tanto como problema, sino como objeto de soluciones y más aún, de satisfacción de necesidades, sin basarse en la deficiencia ni poniendo el acento en la dificultad o en las limitaciones por trastornos o alteraciones (Luque, et al., 2014).

Aceptando que accesibilidad y discapacidad conforman dos adjetivos que deben ir unidos (aplicación efectiva a la realidad sustantiva persona-contexto), en la enseñanza se manifiestan desde un diseño y desarrollo curricular para todos. En este sentido, la adaptación curricular universitaria se aprecia como un medio que proporciona al profesorado una vía de acercamiento, comprensión, apoyo y formación del alumnado con discapacidad y para este, como un vehículo de accesibilidad al currículum y una contribución más a la comprensión de sus circunstancias.

En suma, la adaptación curricular se reconoce como un instrumento que supone estrategias de acercamiento y respuesta a la realidad del alumnado, resultado de un proceso de modificación de uno o más elementos de ese currículum. Por otro lado, sería una estrategia de planificación y actuación para los docentes, haciendo operativa la respuesta a las necesidades educativas (formativas) que puedan presentar los alumnos con discapacidad (Luque y Romero, 2002).

En una universidad que incrementa sus recursos para favorecer la inclusión, se advierten mejoras, modificaciones o instalación de las condiciones de accesibilidad, considerando que la atención al estudiante con discapacidad no puede solamente reducirse a lo físico o arquitectónico, o a la accesibilidad de servicios. El profesorado debe impartir sus clases desde una perspectiva integradora, con una metodología adecuada a las necesidades de su alumnado (con o sin discapacidad), favoreciendo su participación y compensando sus circunstancias, siempre en la frontera del cumplimiento del principio de igualdad de oportunidades.

Ciertamente las barreras disminuyen, a la vez que crece el número de alumnos y alumnas con discapacidad en el ámbito universitario (Nava-Caballero, 2012) pero también, sigue apreciándose la necesidad de compensar las variadas situaciones del alumnado, a través de la gestión de ayudas técnicas, becas, programas de formación y sensibilización, voluntariado, a lo que ha contribuido de forma relevante los Servicios de Apoyo al Alumnado Universitario con Discapacidad. Todo ello, con ser importante y necesario, no es suficiente. En efecto, no serán efectivas las reales posibilidades de aprendizaje y desarrollo de los estudiantes con discapacidad, si no se elimina en el profesorado y otros actores de la comunidad educativa, todo tipo de actitudes paternalistas y sobreprotectoras, así como el pesimismo con relación a las posibilidades de aprendizaje y desarrollo de los estudiantes con alguna discapacidad (Ilizástigui, 2010).

A nuestro juicio, se hace evidente que la comunidad universitaria debe reflexionar en torno a la actitud hacia el estudiantado con discapacidad. En la medida que esta actitud no avance hacia un verdadero apoyo social, seguirán existiendo dificultades de acceso, en la formación y participación de estos estudiantes, junto con limitaciones en su desarrollo personal y social (Luque y Luque-Rojas, 2016). En la misma línea, deberá procurarse una integración de la docencia (con su formación continua), con actitudes (sobre valores de ciudadanía democrática), uso de recursos y metodologías, en una búsqueda constante sobre la adecuación y ajuste a las personas y en particular, a quienes más lo precisan, como son los estudiantes con discapacidad.

Finalmente, recordemos que el objetivo que la Universidad ha de perseguir con su alumnado y, con especial énfasis, con quienes presentan discapacidad, es el de aplicar el principio de igualdad de oportunidades, compensando las necesidades educativas, formando a la persona y favoreciendo el acceso y desarrollo de los estudios. De acuerdo a este principio, el estudiante con discapacidad no debe recibir un trato diferenciador respecto a los demás alumnos, solo la satisfacción de sus ne- 
cesidades especiales con una respuesta educativa compensadora dentro del marco de la enseñanza-aprendizaje. Desde este punto de vista, el sistema universitario será entendido como estructura y organización de provisión de servicios al alumnado, considerando todos los aspectos posibles que incidan en la persona del estudiante y desde una concepción de accesibilidad, destinado a la atención y respuesta a sus necesidades. Tal perspectiva busca un equilibrio entre el saber hacer docente y la tenencia y uso de los recursos, aportándose la base para una evolución y rendimiento académico apropiados, tanto para el alumnado en general, como particularmente, aquellos estudiantes en situación de discapacidad.

\section{Referencias}

Alonso, A., \& Díez, E. (2008). Universidad y discapacidad: indicadores de buenas prácticas y estándares de actuación para programas y servicios. Siglo Cero, 39(2), 82-98.

Diez, E., Sánchez, S. (2014). Diseño universal para el aprendizaje como metodología docente para atender a la diversidad en la universidad. Aula Abierta, 43, 87-93.

Escudero, J. M. (2008). Las competencias profesionales y la formación universitaria: Posibilidades y riesgos. Boletín de la RED-U. Revista de Docencia Universitaria núm. monográf. 2. Recuperado de http://www. redu.um.es/Red_U/m2/.

Grau, X. (2004). Tecnología y discapacidad visual. Necesidades tecnológicas y aplicaciones en la vida diaria de las personas con ceguera y deficiencia visual. Madrid. Fundación UCM-ONCE.

Herradón, R., Blanco, J., Pérez-Juste, A. (2009). Experiencias y metodologías en asignaturas B-Learning para la formación y evaluación en competencias generales en ingeniería. La cuestión Universitaria, 5. Cátedra UNESCO de Gestión y Política Universitaria de la Universidad Politécnica de Madrid.

Ilizástigui, L. (2010). Atención Educativa a la Diversidad desde el desarrollo individual e Institucional. Ponencia presentada en la VII Jornadas Internacionales sobre el tratamiento Educativo a la Diversidad. Madrid, 23 y 24 de abril.

Ley Orgánica 4/2007, de 12 de abril, por la que se modifica la Ley Orgánica 6/2001, de 21 de diciembre, de Universidades. $\mathrm{BOE},(89), 89-109$.

Luque, D., Romero, J. F. (2002). Trastornos del desarrollo y adaptación curricular. Málaga. Aljibe.

Luque, D. J., Rodríguez, G., Romero, J. F. (2005). Accesibilidad y Universidad. Un estudio descriptivo. Intervención Psicosocial, 14(2), 209-222.

Luque, D. J., Rodríguez, G. (2006). Necesidades técnicas del alumnado con discapacidad en la Universidad de Málaga. 4 Congreso Nacional de Tecnología Educativa y Atención a la Diversidad (Tecnoneet). Murcia.

Luque, D. J. y Rodríguez, G. (2009). Tecnología de la Información y la Comunicación aplicada al alumnado con discapacidad: Un acercamiento docente. Revista Iberoamericana de Educación, 49(3), 1-8.

Luque-Rojas, M. J., Calvo, E., Moreno, A. y Luque, D. (2009). Tecnología de la Información y Comunicación y Discapacidad Intelectual. Congreso Internacional sobre el Uso y Buenas Prácticas con TIC. Facultad de Ciencias de la Educación. Noviembre 2009. Universidad de Málaga.

Luque, D., Luque-Rojas, M. (2012). Sociedad del conocimiento y alumnado con necesidades educativas especiales: reflexiones para una escuela inclusiva. Revista Digital de Investigación Educativa Conect@2, (6), 59-80.

Luque, D. J., Luque-Rojas, M. J. (2016). Alumnado universitario con discapacidad: accesibilidad y adaptación del currículum. Educar y Orientar, 33(5), 36-39.

Molina, C., González Badía, J. (2006). Universidad y Discapacidad. Guía de Recursos. Madrid. Ediciones Cermi.

Moriña Diez, A., López Gavira, R., Melero Aguilar, N., Cortés Vega, M., \& Molina Romo, V. M. (2013). El profesorado en la universidad ante el alumnado con discapacidad: ¿Tendiendo puentes o levantando muros? REDU. Revista de Docencia Universitaria, $11(3), 423-442$.

Nava-Caballero, E. (2012). El acceso y la integración de los estudiantes con discapacidad en la Universidad de León. Revista Complutense de Educación, 23(2), 293-316.

Observatorio Universidad y Discapacidad (2012). Guía para implementar el Universal Instructional Design - UID (Diseño Instruccional Universal) en la universidad. Barcelona. Fundación ONCE. Universitat Politécnica de Catalunya.

Organización de las Naciones Unidas para la Educación, la Ciencia y la Cultura (2005). Hacia las Sociedades del Conocimiento. Informe Mundial de la Unesco. París. UNESCO.

Pegalajar-Palomino, M., \& Colmenero-Ruiz, M. J. (2014). Inteligencia emocional en alumnado de Educación Secundaria en contextos multiculturales. Electronic Journal of Research in Educational Psychology, 12(33), 325-342.

Peralta, A. (2007). Libro Blanco sobre universidad y discapacidad. Madrid. Real Patronato sobre Discapacidad.

Perrenoud, P. (2008). Construir las competencias, ¿es darle la espalda a los saberes? Boletín de la RED-U. Extra. 2. Revista de Docencia Universitaria núm. monográf. 2. Recuperado de http://www.redu.um.es/ Red_U/m2/.

Real Decreto Legislativo 1/2013, de 29 de noviembre, por el que se aprueba el Texto Refundido de la Ley General de derechos de las personas con discapacidad y de su inclusión social. BOE, (289), 95635-95673.

Rivero Hernández, D. (2014). Las TIC como instrumento de inclusión de los estudiantes con discapacidad en la universidad española. Cátedra Telefónica-UNED de Responsabilidad Corporativa y Sostenibilidad. Serie Cuadernos de investigación, 2, 5-47.

Rodríguez G., Luque, D. J. (2006). Adaptación Curricular en el alumnado universitario con discapacidad. En J. Uriarte y P. Martín: Necesidades Educativas Especiales, Contextos desfavorecidos y Apoyo social. Bilbao. Psicoex.

Rodríguez-Martín, A., Álvarez-Arregui, E. (2014). Estudiantes con discapacidad en la Universidad. Un estudio sobre su inclusión. Revista Complutense de Educación, 25(2), 457-479.

Rose, D., Meyer, A. (2000). Universal Design for Learning. Journal of Special Education Technology, 15(1), 67-70. 
Rose, D., Meyer, A., Hitchcock, C. (2005). The Universally designed classroom: Accessible Curriculum and digital technologies. Cambridge, MA: Harvard EducationPress.

Sánchez-Montoya, R. (2002). El papel de las nuevas tecnologías en la estimulación de las inteligencias de las personas con necesidades educativas especiales. Congreso Nacional de Tecnología Educativa y Atención a la Diversidad (Tecnonet). Murcia.

Sánchez-Montoya, R. (2007). Capacidades visibles, tecnologías invisibles: Perspectivas y estudio de casos. Comunicación y Pedagogía: Nuevas Tecnologías y Recursos Didácticos. (220), 32-38.

Sapon-Shevin, M. (2013). La inclusión real: Una perspectiva de justicia social [Versión en castellano]. Revista de investigación en Educación, 11(3), 71-85.

Suriá-Martínez, R. S. (2011). Percepción del profesorado sobre su capacitación en el uso de las TICS como instrumento de apoyo para la integración del alumnado con discapacidad. Profesorado. Revista de Currículum y Formación de Profesorado, 15(2), 299-314.

Victoria, J. A. (2013). Hacia un modelo de atención a la discapacidad basado en los derechos humanos. Boletín Mexicano de Derecho Comparado, (138), 1093-1109.

Zabalza, M. A. (2003). Competencias docentes del profesorado universitario. Calidad y desarrollo profesional. Madrid. Narcea.

\section{Anexos}

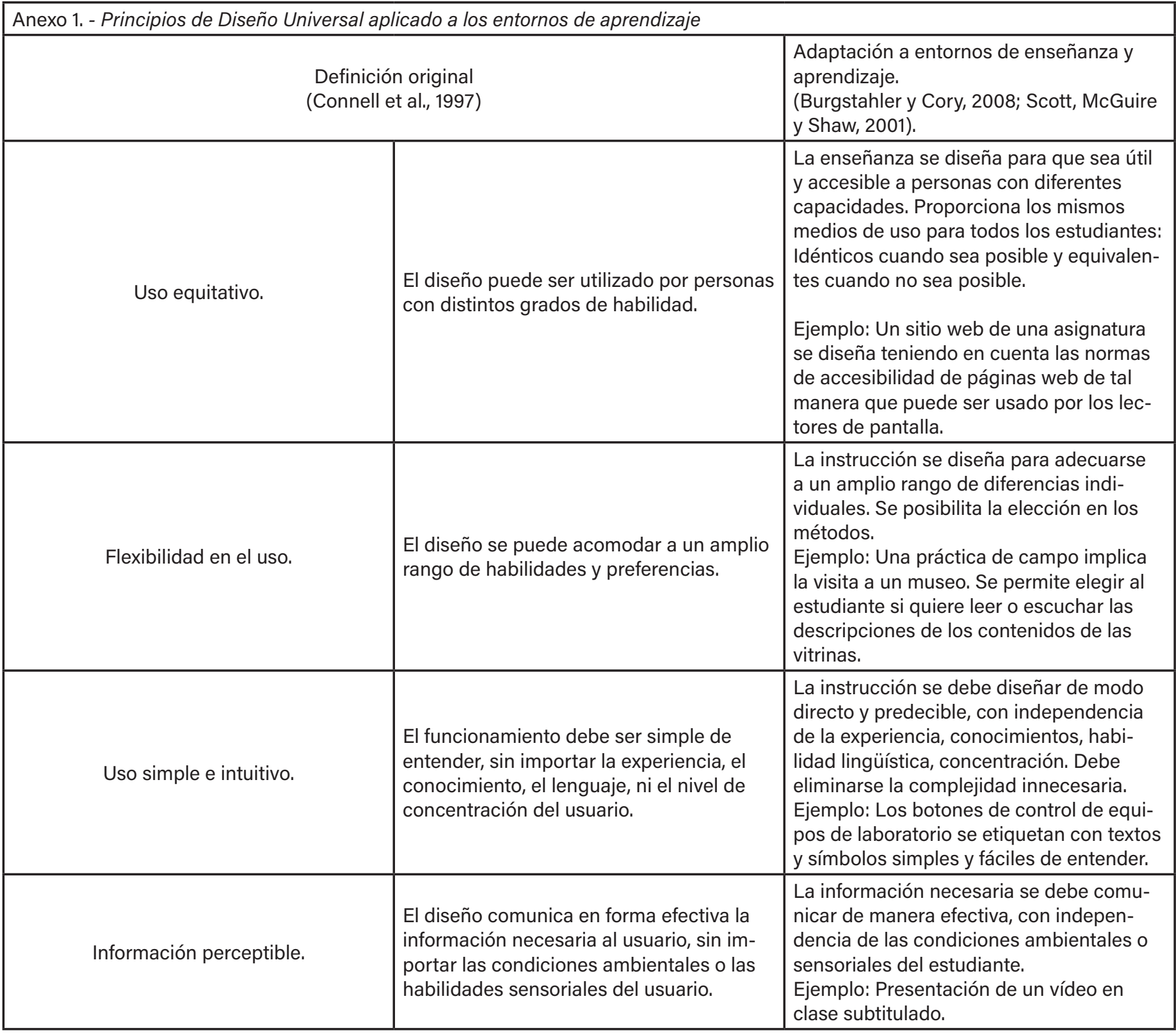




\begin{tabular}{|c|c|c|}
\hline Tolerancia al error. & $\begin{array}{l}\text { El diseño minimiza las consecuencias } \\
\text { adversas de los accidentes o acciones no } \\
\text { intencionadas. }\end{array}$ & $\begin{array}{l}\text { La instrucción anticipa la variabilidad en } \\
\text { los ritmos de aprendizaje y las habilidades } \\
\text { previas requeridas. } \\
\text { Ejemplo: Un entorno virtual de apren- } \\
\text { dizaje proporciona ayudas y guías para } \\
\text { situaciones de respuesta errónea o } \\
\text { inapropiada. }\end{array}$ \\
\hline Mínimo esfuerzo físico-cognitivo. & $\begin{array}{l}\text { El diseño puede ser usado en forma efi- } \\
\text { ciente y cómoda con un mínimo de fatiga } \\
\text { física. }\end{array}$ & $\begin{array}{l}\text { La instrucción ha de minimizar el esfuerzo } \\
\text { físico-cognitivo innecesario. } \\
\text { Ejemplo: Las puertas de la clase se abren } \\
\text { automáticamente. Las páginas-web se } \\
\text { diseñan sin demasiados niveles de anida- } \\
\text { miento y teniendo en cuenta criterios de } \\
\text { usabilidad. }\end{array}$ \\
\hline Espacios y tamaños adecuados. & $\begin{array}{l}\text { Deben proporcionarse espacios y tama- } \\
\text { ños y tamaños apropiados para el acer- } \\
\text { camiento, el uso y la manipulación, sin } \\
\text { importar el tamaño del cuerpo, la postura } \\
\text { o la movilidad del usuario. }\end{array}$ & $\begin{array}{l}\text { La instrucción se diseña teniendo en } \\
\text { cuenta los tamaños y espacios para llegar, } \\
\text { alcanzar, manipular y utilizar, con inde- } \\
\text { pendencia del tamaño de cuerpo, postura, } \\
\text { movilidad y necesidades comunicativas. } \\
\text { Ejemplo: Un laboratorio tiene espacio sufi- } \\
\text { ciente para diestros y zurdos o para quien } \\
\text { quiera trabajar sentado o de pie. }\end{array}$ \\
\hline
\end{tabular}

Anexo 2. - Algunos términos de aplicación y de reflexión docentes

Discapacidad: es una situación que resulta de la interacción entre las personas con deficiencias previsiblemente permanentes y cualquier tipo de barreras que limiten o impidan su participación plena y efectiva en la sociedad, en igualdad de condiciones con las demás.

Igualdad de oportunidades: es la ausencia de toda discriminación, directa o indirecta, por motivo de o por razón de discapacidad, incluida cualquier distinción, exclusión o restricción que tenga el propósito o el efecto de obstaculizar o dejar sin efecto el reconocimiento, goce o ejercicio en igualdad de condiciones por las personas con discapacidad, de todos los derechos humanos y libertades fundamentales en los ámbitos político, económico, social, laboral, cultural, civil o de otro tipo. Asimismo, se entiende por igualdad de oportunidades la adopción de medidas de acción positiva.

Normalización: es el principio en virtud del cual las personas con discapacidad deben poder llevar una vida en igualdad de condiciones, accediendo a los mismos lugares, ámbitos, bienes y servicios que están a disposición de cualquier otra persona

Inclusión social: es el principio en virtud del cual la sociedad promueve valores compartidos orientados al bien común y a la cohesión social, permitiendo que todas las personas con discapacidad tengan las oportunidades y recursos necesarios para participar plenamente en la vida política, económica, social, educativa, laboral y cultural, y para disfrutar de unas condiciones de vida en igualdad con los demás.

Accesibilidad universal: es la condición que deben cumplir los entornos, procesos, bienes, productos y servicios, así como los objetos, instrumentos, herramientas y dispositivos, para ser comprensibles, utilizables y practicables por todas las personas en condiciones de seguridad y comodidad y de la forma más autónoma y natural posible. Presupone la estrategia de «diseño universal o diseño para todas las personas», y se entiende sin perjuicio de los ajustes razonables que deban adoptarse.

Diseño universal o diseño para todas las personas: es la actividad por la que se conciben o proyectan desde el origen, y siempre que ello sea posible, entornos, procesos, bienes, productos, servicios, objetos, instrumentos, programas, dispositivos o herramientas, de tal forma que puedan ser utilizados por todas las personas, en la mayor extensión posible, sin necesidad de adaptación ni diseño especializado. El «diseño universal o diseño para todas las personas» no excluirá los productos de apoyo para grupos particulares de personas con discapacidad, cuando lo necesiten.

Medidas de acción positiva: son aquellas de carácter específico consistentes en evitar o compensar las desventajas derivadas de la discapacidad y destinadas a acelerar o lograr la igualdad de hecho de las personas con discapacidad y su participación plena en los ámbitos de la vida política, económica, social, educativa, laboral y cultural, atendiendo a los diferentes tipos y grados de discapacidad.

Vida independiente: es la situación en la que la persona con discapacidad ejerce el poder de decisión sobre su propia existencia y participa activamente en la vida de su comunidad, conforme al derecho al libre desarrollo de la personalidad.

Ajustes razonables: son las modificaciones y adaptaciones necesarias y adecuadas del ambiente físico, social y actitudinal a las necesidades específicas de las personas con discapacidad que no impongan una carga desproporcionada o indebida, cuando se requieran en un caso particular de manera eficaz y práctica, para facilitar la accesibilidad y la participación y para garantizar a las personas con discapacidad el goce o ejercicio, en igualdad de condiciones con las demás, de todos los derechos.

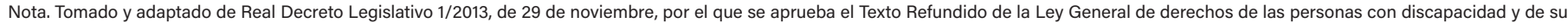
inclusión social. BOE, (289), 95635-95673. 